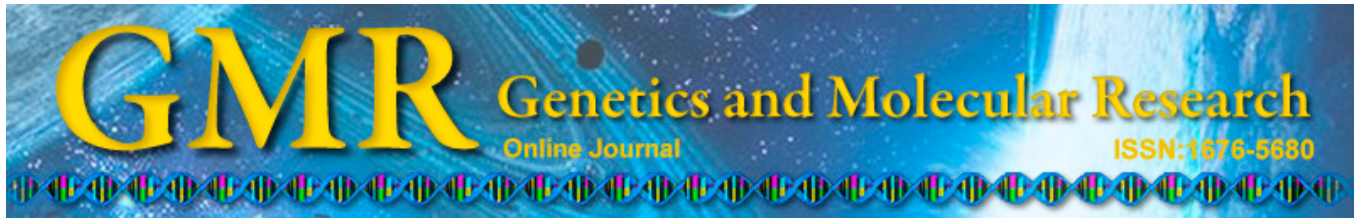

\title{
Cloning and expression analysis of pepper chlorophyll catabolite reductase gene CaRCCR
}

\author{
H.-J. Xiao ${ }^{1,2}$, J.-H. Jin ${ }^{1,2}$, W.-G. Chai ${ }^{3}$ and Z.-H. Gong ${ }^{1,2}$ \\ ${ }^{1}$ College of Horticulture, Northwest A\&F University, Yangling, \\ Shaanxi, China \\ ${ }^{2}$ State Key Laboratory of Crop Stress Biology in Arid Areas, \\ Northwest A\&F University, Yangling, Shaanxi, China \\ ${ }^{3}$ Institute of Vegetables, Hangzhou Academy of Agricultural Sciences, \\ Hangzhou, Zhejiang, China
}

Corresponding author; Z.-H. Gong

E-mail: zhgong@nwsuaf.edu.cn

Genet. Mol. Res. 14 (1): 368-379 (2015)

Received January 2, 2014

Accepted April 26, 2014

Published January 23, 2015

DOI http://dx.doi.org/10.4238/2015.January.23.10

\begin{abstract}
Opening the porphyrin macrocycle of pheophorbide $a$ and forming the primary fluorescent chlorophyll catabolites are key steps in the chlorophyll catabolism pathway. These steps are catalyzed by pheophorbide $a$ oxygenase and red chlorophyll catabolite reductase (RCCR). In this study, a novel RCCR gene, CaRCCR, was isolated from the pepper (Capsicum annuum L.). The full-length CaRCCR complementary DNA is comprised of $1173 \mathrm{bp}$, contains an open reading frame of $945 \mathrm{bp}$, and encodes a 314-amino acid protein. This deduced protein belongs to the ferredoxin-dependent bilin reductase family. Amino acid sequence alignment showed that $\mathrm{CaRCCR}$ shared a high homology to other higher plant RCCR proteins. CaRCCR expression, as determined by quantitative real-time polymerase chain reaction, was higher in the leaves than the roots, stems, flowers, and immature fruits. CaRCCR expression was almost constant during
\end{abstract}


all phases of leaf development. It was upregulated by abscisic acid, methyl jasmonate, and salicylic acid. Moreover, CaRCCR was induced by high salinity and drought stress treatments; it was also slightly regulated by Phytophthora capsici. Taken together, these results suggest that $C a R C C R$ is involved in defense responses to various stresses.

Key words: Capsicum annuum L.; CaRCCR gene; Expression analysis; Cloning; Stress response

\section{INTRODUCTION}

Leaf senescence is the final stage of leaf development, ultimately leading to the death of the whole leaf. It is governed by developmental age, but it can also be stimulated by diverse environmental factors. Such environmental factors include plant hormones, drought, salinity, extreme temperature, darkness, nutrient limitation, wounding, and pathogen infection (Lim et al., 2007; Guo and Gan, 2012). Early leaf senescence can affect the yield of plants under these adverse environmental conditions. Therefore, studying leaf senescence will not only strengthen our comprehending of the basic biological process, but it may also lead to the discovery of methods that can delay leaf senescence and improve the agricultural traits of a vegetable crop. The pepper (Capsicum annuиm L.) is an important vegetable crop; it is extensively cultivated throughout the world. In recent years, prematurity of pepper plants caused by various environmental stresses is a universal phenomenon. It is important to research leaf senescence in the pepper.

Loss of green coloration, induced by the degradation of chlorophyll, is the most obvious sign of leaf senescence and widely used for senescence quantification. In recent years, a pathway of chlorophyll catabolism during senescence has been well established (Hörtensteiner, 1999; Kräutler, 2008; Schelbert et al., 2009; Hörtensteiner and Kräutler, 2011; Sakuraba et al., 2012). The pathway starts with the removal of phytol by chlorophyllase (Willstätter and Stoll, 1913), followed by the removal of $\mathrm{Mg}^{2+}$ by Mg-dechelatase (Suzuki et al., 2005) to produce pheophorbide (pheide) $a$. The porphyrin macrocycle of the pheide $a$ is oxygenolytically cleaved by pheophorbide $a$ oxygenase (PAO) (Hörtensteiner et al., 1998), thus forming a red chlorophyll catabolite (RCC) that is subsequently catalyzed to the primary fluorescent chlorophyll catabolites (pFCCs) by RCC reductase (RCCR) (Mühlecker et al., 1997; Rodoni et al., 1997). These chlorophyll degradation steps occur in chloroplasts; then, the resultant $\mathrm{pFCC}$ is transported to the vacuoles ( $\mathrm{Lu}$ et al., 1998; Tommasini et al., 1998), where pFCC is spontaneously converted into nonfluorescent chlorophyll catabolites in an acidic environment (Oberhuber et al., 2003). The key steps in this catabolism pathway are the ring-opening reaction catalyzed by PAO and RCC-mediated reduction. During these steps, chlorophyll catabolites lose their color and phototoxicity (Pružinská et al., 2005; Sugishima et al., 2009). RCCR strongly participates in chlorophyll degradation and leaf senescence; therefore, it is very important for understanding the molecular mechanism of the RCCR gene in higher plants.

RCCR is a soluble protein and belongs to the ferredoxin-dependent bilin reductase (FDBR) family. RCCR catalyzes the ferredoxin-dependent reduction of the $\mathrm{C} 20 / \mathrm{C} 1$ double bond of RCC to produce pFCC in an intriguing stereospecific manner (Hörtensteiner, 2006; Sugishima et al., 2009). Previous studies found that mutants deficient in RCCR accumulated RCC, resulting in the production of excessive reactive oxygen species and, finally, the light- 
independent cell death phenotype (Mach et al., 2001; Prǔzinská et al., 2007). To date, the $R C C R$ gene has been cloned from many higher plants such as Arabidopsis, rice, tomato, pear, cabbage, and so on (Mach et al., 2001; Tang et al., 2011; Zhang et al., 2011; Cheng et al., 2012). In Arabidopsis, AtRCCR is a single-copy gene and identical to the Arabidopsis accelerated cell death $1(A C D 2)$ gene. AtRCCR/AtACD2 is constitutively expressed in chloroplasts; however, in young seedlings, it is loosely associated with mitochondria as well (Mach et al., 2001; Yao et al., 2004; Yao and Greenberg, 2006).

Since little is known about the CaRCCR gene during leaf senescence in the pepper, this study was conducted to clone this gene from the pepper plant and analyze its molecular characteristics. To learn more about the expression patterns of CaRCCR, analyses of tissuespecific expression and various stress-induced expression patterns via quantitative real-time polymerase chain reaction (PCR) were performed. This study will provide the basis for further studies on the function of RCCR in the pepper.

\section{MATERIAL AND METHODS}

\section{Plant material and growth conditions}

In this study, the pepper cultivar B12 was used for cloning and expression analysis of the pepper $C a R C C R$ gene. This cultivar, which is an early maturing variety, was selected by the pepper research group in the College of Horticulture, Northwest A\&F University, China. The seeds were treated with warm water $\left(55^{\circ} \mathrm{C}\right)$ for $20 \mathrm{~min}$ and then put in an incubator at $28^{\circ} \mathrm{C}$ to accelerate germination under the dark condition. The seeds were rinsed twice every day until budding. The germinated seeds were sown in pots containing compost. Seedlings were placed in a growth chamber for a 16 -h light $/ 8$-h dark cycle at $25^{\circ} / 21^{\circ} \mathrm{C}$, respectively.

\section{CaRCCR gene expression pattern analysis}

\section{Tissue-specific expression of the CaRCCR gene}

To evaluate the CaRCCR gene expression levels in different tissues, samples were collected from the root, stem, leaf, flower, and immature fruit of untreated B12 pepper cultivars. To examine the CaRCCR expression pattern during leaf development, leaves were harvested at the young, fully expanded, and senescent stages. All samples were frozen in liquid nitrogen and maintained at $-80^{\circ} \mathrm{C}$ prior to RNA extraction.

\section{Stress treatments}

The signaling molecule, and abiotic and biotic stresses were used to treat the 6-leaf stage pepper plants. For the plant signaling molecule treatments, 3 plant hormones were applied, and the pepper plant leaves were sprayed with $0.57 \mathrm{mM}$ abscisic acid (ABA), $5 \mathrm{mM}$ salicylic acid (SA), and $1 \mathrm{M}$ methyl jasmonate (MeJA), which were dissolved in $0.05 \%(\mathrm{v} / \mathrm{v})$ ethanol. The control plants were treated with distilled water that contained $0.05 \%(\mathrm{v} / \mathrm{v})$ ethanol. After 0-, 2-, 4-, 8-, 12-, and 24-h treatments, the pepper leaves were sampled. For salt and drought stress treatments, the pepper plants were uprooted from the soil, and their roots 
were soaked in $400 \mathrm{mM}$ sodium chloride $(\mathrm{NaCl})$ and $400 \mathrm{mM}$ mannitol, respectively (Wang et al., 2013b). The control seedlings were treated with sterile water. After the 0-, 2-, 4-, 8-, 12-, and 24-h treatments, the pepper leaves were collected. For the fungal pathogen treatment, pepper plants were inoculated with Phytophthora capsici (HX-9 strain) by the root-drenching method; the control plants were treated with sterile distilled water (Wang et al., 2013a). The inoculated and control plants were grown in a growth chamber at $28^{\circ} \mathrm{C}$ under a $16-\mathrm{h}$ light $/ 8-\mathrm{h}$ dark photoperiod cycle with $60 \%$ relative humidity. The samples were collected at $0-, 3-, 6-$, 12-, 24-, 48-, 72-, and 96-h intervals.

All plants that were treated with the different chemicals and stress elicitors, and the control plants were sampled at the above mentioned times, immediately frozen in liquid nitrogen, and maintained at $-80^{\circ} \mathrm{C}$ prior to RNA extraction.

\section{Cloning and sequence analysis of the $C a R C C R$ gene}

For cloning of the CaRCCR gene, total RNA was extracted from green mature leaves using the TRIzol (Invitrogen) method, and first-strand complementary DNA (cDNA) synthesis was performed using the Smart RACE cDNA Amplification Kit (Clontech). The reported nucleotide sequence of Solanum lycopersicum RCCR (accession No. XM004234620) was used as the query probe to retrieve homologous expressed sequence tags (ESTs) of the pepper in GenBank using the BLASTN analysis. Three overlapping ESTs of the pepper (accession Nos. GD090771, GD097572, and GD089113) were chosen for assembly in a splicing fragment without a complete open reading frame (ORF). To confirm the authenticity of an assembled sequence in the pepper, a pair of primers, EST-RCCR-F and EST-RCCR-R, was utilized (Table 1). The design of both primers was based on the splicing sequence. The PCR procedure was as follows: $94^{\circ} \mathrm{C}$ for $5 \mathrm{~min} ; 35$ cycles of $94^{\circ} \mathrm{C}$ for $30 \mathrm{~s}, 60^{\circ} \mathrm{C}$ for $30 \mathrm{~s}$, and $72^{\circ} \mathrm{C}$ for $1.0 \mathrm{~min}$; and, finally, an extension of $72^{\circ} \mathrm{C}$ for $10 \mathrm{~min}$. The corresponding sequence was obtained, subcloned into the pMD19-T vector (TaKaRa), and sequenced.

To isolate a full-length cDNA of the putative CaRCCR gene with complete 5'- and 3'-regions, the RACE method was used. The gene-specific primers (5'-GSP, 5'-NGSP, 3'-GSP, and 3 '-NGSP) were designed according to the partial cDNA sequence (Table 1). The primers of 5'-GSP (external primer) and 5'-NGSP (internal primer) were used to isolate 5'-region sequences; the primers of the 3'-GSP (external primer) and 3'-NGSP (internal primer) were used for isolation of 3'-end sequences. The nested PCR was performed via 5'-RACE and 3'-RACE. The amplified PCR products were ligated into the pMD19-T vector and sequenced. Finally, all acquired sequences were assembled into a sequence with a complete ORF using the Contig Expression Software and the BLAST software online.

The cDNA and protein sequences of the putative $C a \mathrm{RCCR}$ were analyzed by the DNAMAN software 5.2.2 and the BLAST software online (http://blast.ncbi.nlm.nih.gov/ Blast.cgi). Prediction of the putative signal peptide subcellular localization, chloroplast transit peptides, and its cleavage site were carried out using the CBS prediction servers online program (http://www.cbs.dtu.dk/services/). Isoelectric point (pI) and molecular weight (MW) of the putative protein were analyzed by the $\mathrm{pI} / \mathrm{MW}$ program (http://www.expasy.org/), and its secondary structure was predicted by the scratch protein predictor program online (http:// scratch.proteomics.ics.uci.edu). The multiple sequence alignments of $\mathrm{CaRCCR}$ and other RCCR proteins were performed by the DNAMAN software. The phylogenetic tree was constructed using the MEGA5.05 program with the neighbor-joining method. 


\section{RNA isolation}

Total RNA was extracted from different pepper tissues (without treatment) and leaves (with different treatments, as mentioned above) using the TRIzol method. Contaminated genomic DNA was digested by RNase-free DNase I (Promega). The concentration and purity of total RNA were determined spectrophotometrically using a NanoDrop instrument (Thermo Scientific NanoDrop 2000C Technologies, Wilmington, DE, USA). The first-strand cDNA was synthesized according to the instructions of the PrimeScript ${ }^{\mathrm{TM}}$ Kit (TaKaRa).

\section{Quantitative real-time PCR analysis}

In order to identify the expression patterns of $C a R C C R$ in the different pepper tissues without treatment and the leaves under various stresses, quantitative real-time PCR was performed using the SYBR ${ }^{\circledR}$ Premix Ex Taq ${ }^{\mathrm{TM}} \mathrm{II}(\mathrm{TaKaRa})$. The reaction system for real-time reverse transcription (RT) PCR analysis was a $20-\mu \mathrm{L}$ reaction volume containing $10.0 \mu \mathrm{L}$ SYBR $^{\circledR}$ Premix Ex Taq ${ }^{\mathrm{TM}}$ II, $2.0 \mu \mathrm{L}$ diluted cDNA, and $0.8 \mu \mathrm{L}$ forward and reverse primers. The amplification was completed with the cycling parameters as follows: $95^{\circ} \mathrm{C}$ for $1 \mathrm{~min}$, followed by $95^{\circ} \mathrm{C}$ for $10 \mathrm{~s}, 57^{\circ} \mathrm{C}$ for $20 \mathrm{~s}$, and $72^{\circ} \mathrm{C}$ for $20 \mathrm{~s}$ for 40 cycles. The gene of $U B I 3$ (accession No. AY486137.1) was used as the internal control (reference gene) in this study (Table 1). The relative expression levels of the gene were calculated with the comparative threshold method of Livak and Schmittgen (2001). All treatments were performed in 3 independent biological replicates.

\section{Primers used in this study}

Primers used for cloning and quantitative real-time PCR analysis of the CaRCCR gene are listed in Table 1.

\begin{tabular}{ll}
\multicolumn{2}{|c}{ Table 1. Primer sequences used in this study. } \\
\hline Primer & \\
\hline Cloning of CaRCCR gene & Sequence (5'-3') \\
EST-RCCR-F & \\
EST-RCCR-R & TGGTGGAACTTGTATCTACTCTG \\
5'-RACE-GSP & TATTTATTCGATCCCTTTTAGC \\
5'-RACE-NGSP & CTCTGCTTGTCTAACTGTGTGTCTTC \\
3'-RACE-GSP & CAAGTATGAAATCAACCTGAGAAGC \\
3'-RACE-NGSP & GATTTCATACTTGGTAGTTGGGTC \\
Quantitative real-time PCR & GAAGACACACAGTTAGACAAGCAGAG \\
RT-RCCR-F & \\
RT-RCCR-R & TGGTGGAACTTGTATCTACTCTGG \\
CaUBI3F & CTACCAAGTATGAAATCAACCTGAG \\
CaUBI3R & TGTCCATCTGCTCTCTGTTG \\
\hline
\end{tabular}

\section{RESULTS}

\section{Cloning and sequence analysis of $\mathrm{CaRCCR}$}

The full-length cDNA, designated $C a R C C R$, was obtained using the bioinformatic 
analysis and RACE techniques. The transcript consisted of 1173 nucleotides, including a 9-bp 5'-untranslated region (UTR), 945-bp ORF, and 219-bp 3'-UTR (GenBank accession No. KC176711; Figure 1). CaRCCR was predicted to encode 314 amino acids, the theoretical molecular mass of the encoded protein was $35.2 \mathrm{kDa}$, and the calculated pI was 5.45. Structural analysis revealed that $\mathrm{CaRCCR}$ belonged to the FDBR family and contained 2 conserved acidic residue sites (Glu151 and Asp288), which are involved in catalysis and/or substrate binding. Subcellular localization analysis demonstrated that $\mathrm{CaRCCR}$ was located in the chloroplast. Furthermore, sequence analysis indicated that the deduced $\mathrm{CaRCCR}$ protein contains a chloroplast transit peptide with 37-amino acid residues; its cleavage site was located between $\mathrm{P}_{37}$ and $\mathrm{M}_{38}$. The deduced $\mathrm{CaRCCR}$ did not have a signal peptide region or transmembrane helix.

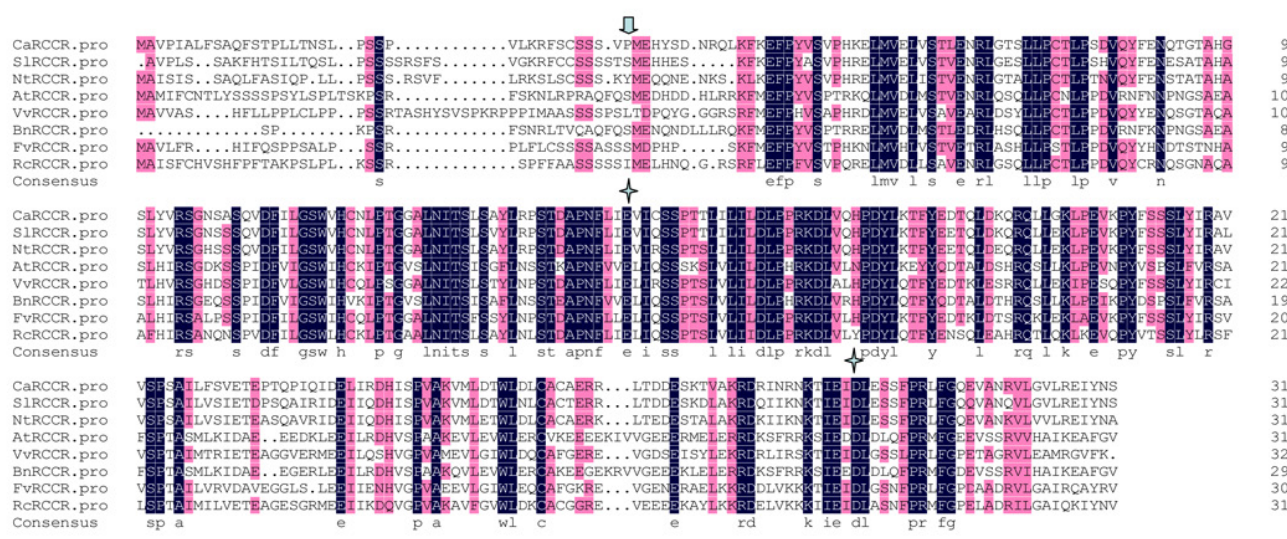

Figure 1. Multiple-sequence alignment of the CaRCCR protein and RCCR proteins from other plants using the DNAMAN software. Blue and gray shaded regions reflect 100 and $80 \%$ sequence conservation, respectively. The arrow indicates the cleavage site of the chloroplast peptide, and the asterisks indicate 2 conserved acidic residue sites (Glu151 and Asp288). In addition to CaRCCR, other amino acid sequences included in this alignment were Solanum lycopersicum RCCR (CAJ80766.1), Nicotiana tabacum RCCR (ABY19386.1), Vitis vinifera RCCR (XP_002277744.1), Ricinus communis RCCR (XP_002523576.1), Fragaria vesca subsp vesca RCCR (XP_004307021.1), Brassica napus RCCR (CAJ80767.1), and Arabidopsis thaliana RCCR (NP_195417.1).

The deduced $\mathrm{CaRCCR}$ amino acid sequence showed high homology to other plant RCCR sequences via multiple alignment using the DNAMAN software (Figure 1). The percent identities of CaRCCR relative to Solanum lycopersicum RCCR (CAJ80766.1), Nicotiana tabacum RCCR (ABY19386.1), Vitis vinifera RCCR (XP_002277744.1), Ricinus communis RCCR (XP 002523576.1), Fragaria vesca subsp vesca RCCR (XP 004307021.1), Brassica napus RCCR (CAJ80767.1), and Arabidopsis thaliana RCCR (NP_195417.1) were 82, 79, $60,59,59,53$, and $50 \%$, respectively.

A phylogenetic tree constructed using the MEGA5.05 software was used to investigate the evolutionary relationships of the $\mathrm{CaRCCR}$ amino acid sequence in comparison to the RCCR proteins of other plants. Two groups were formed using the 13 RCCR protein sequences from $C$. annuum L., S. lycopersicum, $N$. tabacum, $R$. communis, $V$. vinifera, B. napus, A. thaliana, F. vesca subsp vesca, Medicago truncatula, Theobroma cacao, Glycine max, Citrullus lanatus subsp vulgaris, and Cucumis sativus (Figure 2). CaRCCR clustered in the first group, which included SlRCCR, NtRCCR, CsRCCR, RcRCCR, and so on. Phylogenetic 
analysis showed that CaRCCR was more closely related to SlRCCR and NtRCCR than the RCCR proteins of other plants.

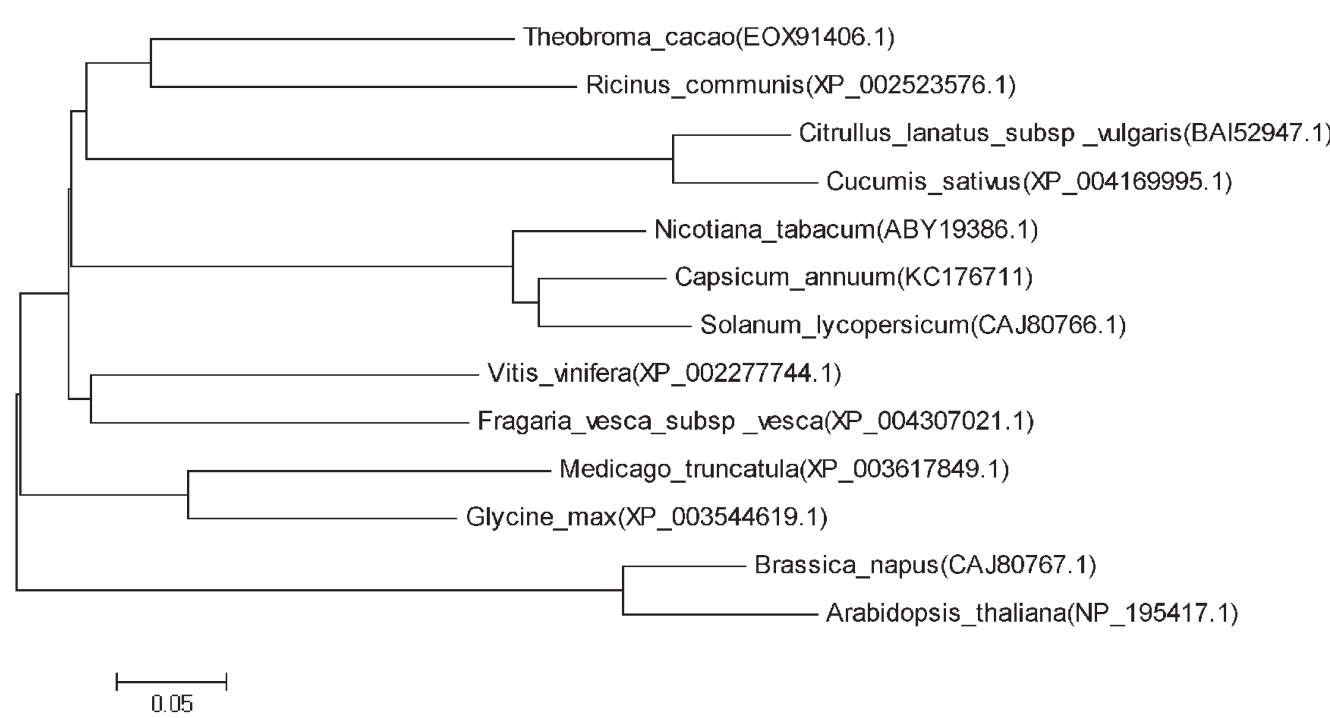

Figure 2. Phylogenetic analysis of the $C a$ RCCR protein and RCCR proteins of other plant species. The phylogenetic tree was constructed by the neighbor-joining method using MEGA 5.05. Branches were labeled with the names and GenBank accession Nos. of the different plant species.

\section{Tissue-specific expression of $C a R C C R$}

In order to investigate the expression levels of the CaRCCR gene in different tissues of B12 pepper cultivar plants, total RNA was extracted from the roots, stems, leaves, flowers, and immature fruits, and quantitative real-time PCR was performed (Figure 3a). CaRCCR transcripts were detected in all of these tissues and found to be higher in the leaves than in the other tissues. During the 3 developmental stages, the transcript level of $C a R C C R$ was almost constant in the young leaves, fully expanded leaves, and senescent leaves (Figure 3b).

\section{Expression analysis of the CaRCCR gene in pepper plants treated with signaling molecules}

To examine whether signal molecules induce $C a R C C R$ expression, 3 phytohormones were used in the pepper leaves. As shown in Figure 4, the expression levels of CaRCCR in the leaves sprayed with ABA, MeJA, and SA were elevated to different levels. ABA treatment could quickly induce expression of the $C a R C C R$ gene within the first $4 \mathrm{~h}$ after treatment. In comparison to the control, the highest transcript levels (4.67-fold) of CaRCCR were detected at $8 \mathrm{~h}$. Then, the expression of $C a R C C R$ decreased gradually after $8 \mathrm{~h}$. Spraying pepper plants with MeJA caused rapid upregulation of $C a R C C R$ at $2 \mathrm{~h}$ to the highest observed expression level (2.85-fold) and then quickly declined at $4 \mathrm{~h}$. However, CaRCCR transcripts increased gradually from 8 to $12 \mathrm{~h}$. In contrast, the treatment of SA induced CaRCCR transcript abun- 
dance at $2 \mathrm{~h}$; CaRCCR expression peaked by $4 \mathrm{~h}$, which was 4.05 -fold greater than that of the control, and was then gradually downregulated. Interestingly, there was a slight upregulation at $12 \mathrm{~h}$. These results indicate that the $C a R C C R$ gene could be induced and upregulated by all 3 signaling molecules tested (i.e., ABA, MeJA, and SA).
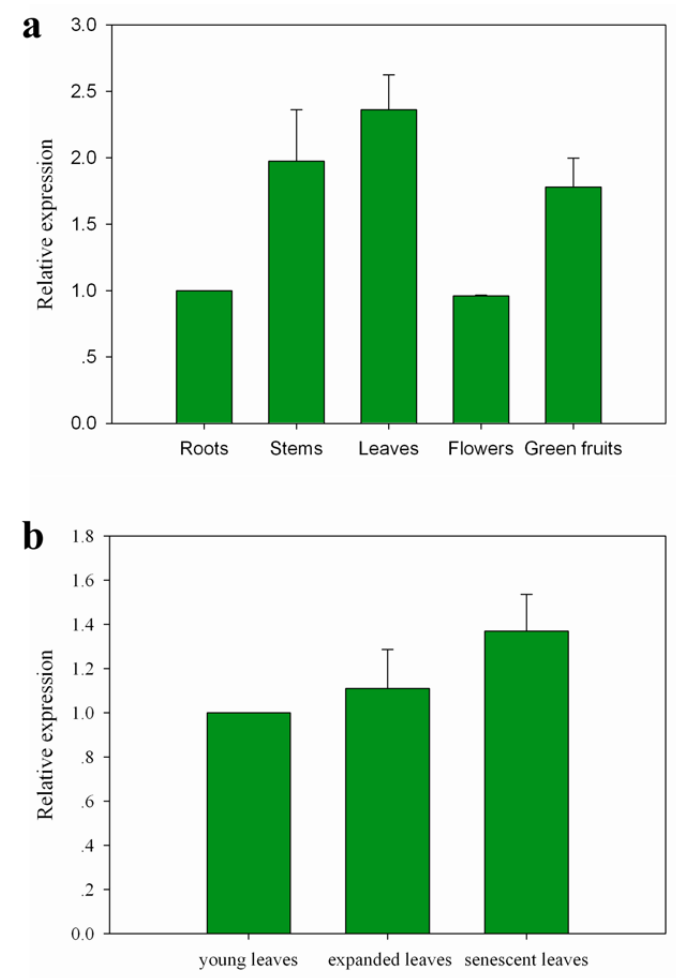

Figure 3. a. Tissue-specific expression of $C a R C C R$ in pepper roots, leaves, stems, flowers, and immature fruits. Relative expression levels of the $C a R C C R$ transcript were determined in different tissues in comparison to that in roots. b. Expression profiles of CaRCCR during the leaf developmental stage. RNA was extracted from the young leaf, fully expanded leaf, and senescent leaf. Error bars represent SD for 3 independent replicates.

\section{Expression patterns of $C a R C C R$ induced by abiotic stresses}

To analyze the expression pattern of the CaRCCR gene in response to abiotic stresses, pepper plants were exposed to $400 \mathrm{mM} \mathrm{NaCl}$ or $400 \mathrm{mM}$ mannitol. The abundance of CaRCCR transcripts was analyzed via quantitative RT-PCR. As shown in Figure 5a, the CaRCCR expression level in plants treated with $400 \mathrm{mM} \mathrm{NaCl}$ exhibited little change within the first $4 \mathrm{~h}$ after treatment; it then began to increase gradually at $8 \mathrm{~h}$ and peaked (2.51-fold) at $12 \mathrm{~h}$ when compared to the control $(0 \mathrm{~h})$. For the drought treatment, the transcript level of $C a R C C R$ was induced in the pepper plants (Figure 5b). The increased expression was shown at $2 \mathrm{~h}$ (1.75-fold), upregulated strongly at $12 \mathrm{~h}$ (3.95-fold), and maintained a relatively steady level from 12 to $24 \mathrm{~h}$ (4.03-fold). However, there was a slight increase in downregulation at $8 \mathrm{~h}$. The above-mentioned results indicate that the CaRCCR gene could be induced and upregulated under abiotic stresses, including high salinity and drought stress. 


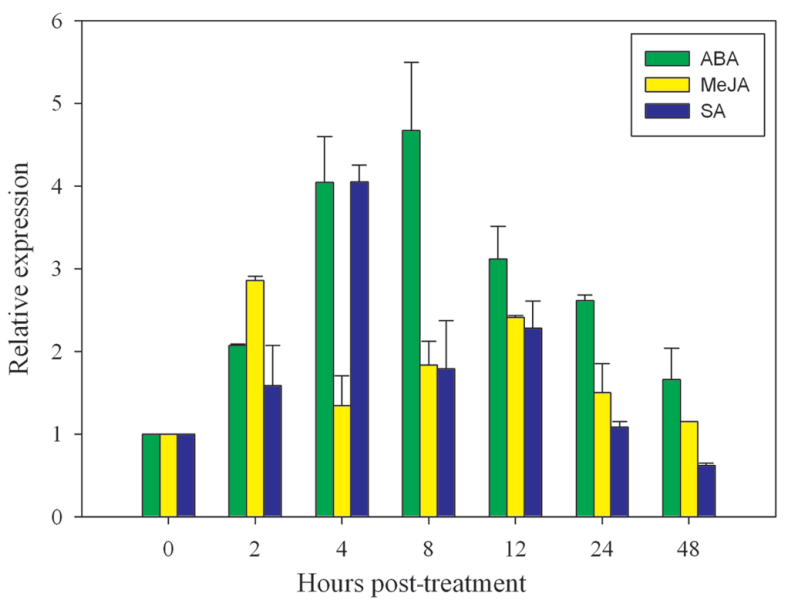

Figure 4. $C a R C C R$ expression patterns in pepper leaves treated with various phytohormones, including abscisic acid (ABA), methyl jasmonate (MeJA), and salicylic acid (SA). The relative transcriptional expression of CaRCCR was calculated in phytohormone-treated leaves in comparison to that in the mock controls. Error bars represent SD for 3 independent replicates.
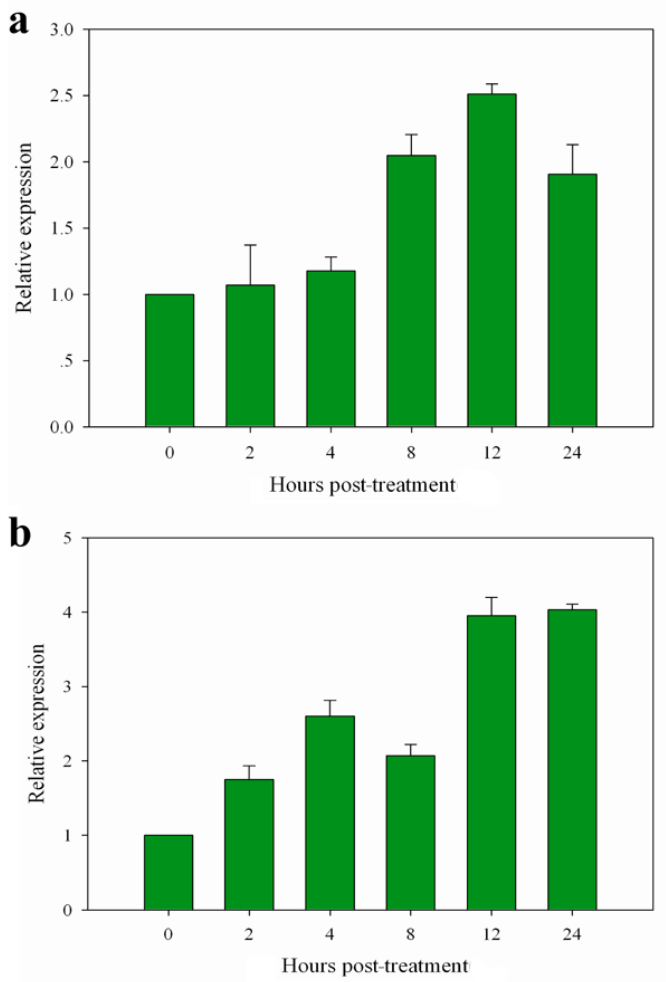

Figure 5. Expression patterns of CaRCCR in pepper leaves treated with abiotic stresses. (a) Salt stress. (b) Drought stress. The relative expression of $C a R C C R$ was calculated in various treated leaves in comparison to that in the mock controls. Error bars represent SD for 3 independent replicates. 


\section{Expression analysis of the CaRCCR gene in response to biotic stresses}

In this study, the expression of $C a R C C R$ slightly increased when the pepper plants were infected with Phytophthora capsici (Figure 6). The transcript level of CaRCCR maintained nearly the same degree at $3 \mathrm{~h}$ and then gradually decreased from 6 to $24 \mathrm{~h}$ when compared to the mock control. The transcript expression level increased rapidly and reached its peak (1.75-fold) at $48 \mathrm{~h}$. Subsequently, the expression level began to decrease at $72 \mathrm{~h}(0.84-$ fold) but slightly increased to 1.28 -fold at $96 \mathrm{~h}$, which was the lowest actual time point in comparison to the control. These results suggest that there are changes in gene expression patterns under $P$. capsici infection.

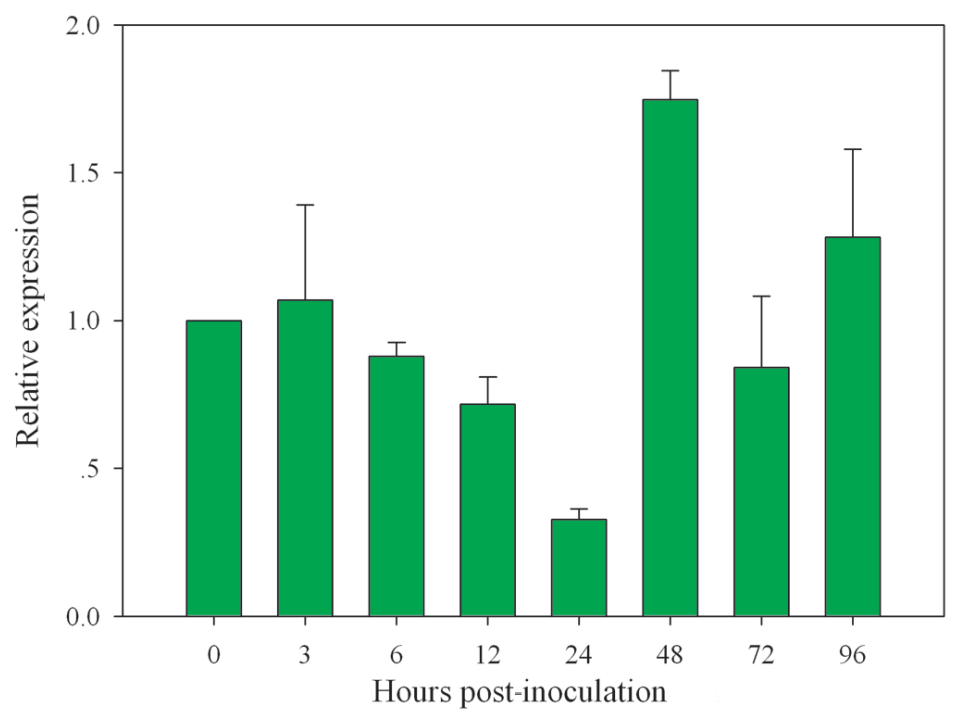

Figure 6. Expression analysis of CaRCCR in pepper leaves after infection with Phytophthora capsici. CaRCCR relative expression was calculated in $P$. capsici-infected leaves in comparison to that in the mock controls. Error bars represent SD for 3 independent replicates.

\section{DISCUSSION}

RCCR catalyzes RCC, the product of the pheide $a$ ring opening to the pFCCs. RCCR is an important constituent of the detoxification of photodynamic chlorophylls and their catabolites. Herein, we identified the CaRCCR gene from the pepper. The predicted CaRCCR amino acid sequence contained the conserved sites (Glu151 and Asp288 in Capsicum annuum L.) that are present in the RCCR protein of other higher plants. Moreover, $\mathrm{CaRCCR}$ shared a high degree of identity with $S / R C C R$ and $N t$ RCCR, and they fall into the same group within the phylogenetic tree. Similar to known reported RCCR proteins, CaRCCR existed as a conserved transit peptide at the $\mathrm{N}$ terminus. These results demonstrate that $\mathrm{CaRCCR}$ should function as an RCCR.

Tissue-specific analysis showed that $C a R C C R$ was expressed in all tissues, such as the 
roots, stems, leaves, flowers, and fruits, and the expression level of CaRCCR in the leaves was higher than that in other tissues. The expression level of CaRCCR in the leaves at different developmental stages exhibited little change. The tissue-specific expression of $C a R C C R$ was similar to the results in rice. It was reported that expression of the $O s R C C R$ gene was detected in all tissues, and expression was the highest in leaves. However, the expression pattern of $C a R C C R$ during leaf development was different than that in rice; in rice, the expression of the OsRCCRI gene was upregulated in naturally senescent leaves (Tang et al., 2011). These results reveal that $R C C R$ is important to the growth and development of all tissues, although its function may be distinct in different species.

Previous studies have shown that chlorophyll degradation is regulated by hormones during the leaf developmental stage and in response to various environmental stresses (Costa et al., 2005; Lim et al., 2007; Bari and Jones, 2009). Chlorophyll degradation of the Chinese cabbage during post-harvest senescence was regulated positively by ethylene and negatively by cytokinin (Zhang et al., 2011). In this study, quantitative real-time PCR analysis indicates that treatment with $\mathrm{ABA}, \mathrm{MeJA}$, or SA was found to increase the expression level of CaRCCR to different extents. These findings suggest that the CaRCCR gene may be involved in ABA-, JA-, and SA-dependent signaling pathways.

Various abiotic and biotic stresses can affect growth and development of pepper plants, causing chlorophyll breakdown, cell death, and, finally, premature senescence. All of these consequences would impact the yield and quality of crop plants. Therefore, it is important to understand the molecular adaptation mechanisms of plants to diverse stresses. Here, using quantitative real-time PCR analysis, we examined the CaRCCR expression pattern in pepper leaves treated with high salinity, drought stress, and P. capsici infection. The expression of the $C a R C C R$ gene was induced under all of the above-mentioned stress conditions. The results showed that CaRCCR may be involved in response to abiotic and biotic stresses in the pepper plant, including high salinity, drought stress, and pathogen infection. These results were different from those of a study in Arabidopsis reporting that $A t R C C R$ expression was rather constant under stress conditions (Prǔzinská et al., 2007). This different expression pattern of RCCR between Arabidopsis and the pepper implies that there are differences in the transcriptional regulation of chlorophyll catabolism in different species.

In summary, the CaRCCR gene was transcribed at various levels in all tissues. In pepper leaves treated with various stresses, including phytohormones, drought, salt, and $P$. capsici infection, CaRCCR was induced, implicating its involvement in abiotic and biotic stresses. Taken together, these data suggest that CaRCCR plays a role in whole plant development and involves the response to various environmental stresses of the pepper plant. In the future, transgenic plants exhibiting overexpression or knockdown of the CaRCCR gene in the pepper should be used to study the detailed function of CaRCCR.

\section{ACKNOWLEDGMENTS}

Research supported by the National Natural Science Foundation of China (\#31272163), "The Twelfth Five-Year" Plan of National Science and Technology in Rural Areas (\#2011BAD12B03), and the Shaanxi Provincial Science and Technology Coordinating Innovative Engineering Project (\#2012KTCL02-09). 


\section{REFERENCES}

Bari R and Jones JD (2009). Role of plant hormones in plant defence responses. Plant Mol. Biol. 69: 473-488.

Cheng YD, Dong Y, Yan HB, Ge WY, et al. (2012). Effects of 1-MCP on chlorophyll degradation pathway-associated genes expression and chloroplast ultrastructure during the peel yellowing of Chinese pear fruits in storage. Food Chem. 135: 415-422.

Costa ML, Civello PM, Chaves AR and Martínez GA (2005). Effect of ethephon and 6-benzylaminopurine on chlorophyll degrading enzymes and a peroxidase-linked chlorophyll bleaching during post-harvest senescence of broccoli (Brassica oleracea L.) at $20^{\circ} \mathrm{C}$. Postharvest Biol. Technol. 35: 191-199.

Guo Y and Gan SS (2012). Convergence and divergence in gene expression profiles induced by leaf senescence and 27 senescence-promoting hormonal, pathological and environmental stress treatments. Plant Cell Environ. 35: 644-655.

Hörtensteiner S (1999). Chlorophyll breakdown in higher plants and algae. Cell Mol. Life Sci. 56: 330-347.

Hörtensteiner S (2006). Chlorophyll degradation during senescence. Annu. Rev. Plant Biol. 57: 55-77.

Hörtensteiner S and Kräutler B (2011). Chlorophyll breakdown in higher plants. Biochim. Biophys. Acta 1807: 977-988.

Hörtensteiner S, Wüthrich KL, Matile P, Ongania KH, et al. (1998). The key step in chlorophyll breakdown in higher plants. Cleavage of pheophorbide a macrocycle by a monooxygenase. J. Biol. Chem. 273: 15335-15339.

Kräutler B (2008). Chlorophyll breakdown and chlorophyll catabolites in leaves and fruit. Photochem. Photobiol. Sci. 7: $1114-1120$.

Lim PO, Kim HJ and Nam HG (2007). Leaf senescence. Annu. Rev. Plant Biol. 58: 115-136.

Livak KJ and Schmittgen TD (2001). Analysis of relative gene expression data using real-time quantitative PCR and the 2(-Delta Delta C(T)) method. Methods 25: 402-408.

Lu YP, Li ZS, Drozdowicz YM, Hörtensteiner S, et al. (1998). AtMRP2, an Arabidopsis ATP binding cassette transporter able to transport glutathione S-conjugates and chlorophyll catabolites: functional comparisons with Atmrp1. Plant Cell 10: 267-282.

Mach JM, Castillo AR, Hoogstraten R and Greenberg JT (2001). The Arabidopsis accelerated cell death gene ACD2 encodes red chlorophyll catabolite reductase and suppresses the spread of disease symptoms. Proc. Natl. Acad. Sci. U S A 98: 771-776.

Mühlecker W, Ongania KH, Kräutler B, Matile P, et al. (1997). Tracking down chlorophyll breakdown in plants: elucidation of the constitution of a "fluorescent" chlorophyll catabolite. Angew. Chem. Int. Edit. 36: 40-404.

Oberhuber M, Berghold J, Breuker K, Hörtensteiner S, et al. (2003). Breakdown of chlorophyll: a nonenzymatic reaction accounts for the formation of the colorless "nonfluorescent" chlorophyll catabolites. Proc. Natl. Acad. Sci. U S A 100: 6910-6915.

Pružinská A, Tanner G, Aubry S, Anders I, et al. (2005). Chlorophyll breakdown in senescent Arabidopsis leaves. Characterization of chlorophyll catabolites and of chlorophyll catabolic enzymes involved in the degreening reaction. Plant Physiol. 139: 52-63.

Prǔzinská A, Anders I, Aubry S, Schenk N, et al. (2007). In vivo participation of red chlorophyll catabolite reductase in chlorophyll breakdown. Plant Cell 19: 369-387.

Rodoni S, Muhlecker W, Anderl M, Kräutler B, et al. (1997). Chlorophyll breakdown in senescent chloroplasts (cleavage of pheophorbide a in two enzymic steps). Plant Physiol. 115: 669-676.

Sakuraba Y, Schelbert S, Park SY, Han SH, et al. (2012). STAY-GREEN and chlorophyll catabolic enzymes interact at lightharvesting complex II for chlorophyll detoxification during leaf senescence in Arabidopsis. Plant Cell 24: 507-518.

Schelbert S, Aubry S, Burla B, Agne B, et al. (2009). Pheophytin pheophorbide hydrolase (pheophytinase) is involved in chlorophyll breakdown during leaf senescence in Arabidopsis. Plant Cell 21: 767-785.

Sugishima M, Kitamori Y, Noguchi M, Kohchi T, et al. (2009). Crystal structure of red chlorophyll catabolite reductase: enlargement of the ferredoxin-dependent bilin reductase family. J. Mol. Biol. 389: 376-387.

Suzuki T, Kunieda T, Murai F, Morioka S, et al. (2005). Mg-dechelation activity in radish cotyledons with artificial and native substrates, Mg-chlorophyllin $a$ and chlorophyllide $a$. Plant Physiol. Biochem. 43: 459-464.

Tang Y, Li M, Chen Y, Wu P, et al. (2011). Knockdown of OsPAO and OSRCCRI cause different plant death phenotypes in rice. J. Plant Physiol. 168: 1952-1959.

Tommasini R, Vogt E, Fromenteau M, Hörtensteiner S, et al. (1998). An ABC-transporter of Arabidopsis thaliana has both glutathione-conjugate and chlorophyll catabolite transport activity. Plant J. 13: 773-780.

Wang JE, Li DW, Zhang YL, Zhao Q, et al. (2013a). Defence responses of pepper (Capsicum annuum L.) infected with incompatible and compatible strains of Phytophthora capsici. Eur. J. Plant Pathol. 136: 625-638.

Wang JE, Liu KK, Li DW, Zhang YL, et al. (2013b). A novel peroxidase CanPOD gene of pepper is involved in defense responses to Phytophtora capsici infection as well as abiotic stress tolerance. Int. J. Mol. Sci. 14: 3158-3177.

Willstätter R and Stoll A (1913). "Die Wirkungen der Chlorophyllase." Untersuchungen über Chlorophyll. Springer, Berlin, Heidelberg, 172-187.

Yao N and Greenberg JT (2006). Arabidopsis ACCELERATED CELL DEATH2 modulates programmed cell death. Plant Cell 18: 397-411.

Yao N, Eisfelder BJ, Marvin J and Greenberg JT (2004). The mitochondrion - an organelle commonly involved in programmed cell death in Arabidopsis thaliana. Plant J. 40: 596-610.

Zhang XL, Zhang ZQ, Li J, Wu LJ, et al. (2011). Correlation of leaf senescence and gene expression/activities of chlorophyll degradation enzymes in harvested Chinese flowering cabbage (Brassica rapa var. parachinensis). J. Plant Physiol. 168: 2081-2087. 